\title{
Communication
}

\section{Identification and characteristics of drug resistance and genotypes of methicillin-resistant Staphylococcus aureus isolated from intensive care units at obstetrics \& gynaecology departments: a retrospective analysis}

\author{
Zhonghua Huo ${ }^{1}$, Binxian $\mathrm{Li}^{1, *, \dagger}$, Xue Meng ${ }^{2}$, Peiyao $\mathrm{Li}^{2}$, Mingcheng $\mathrm{Li}^{2}, *, \dagger$ \\ ${ }^{1}$ Department of Clinical Microbiology, Associated hospital, Beihua University, 132013 Jilin, Jilin, China \\ ${ }^{2}$ Department of Clinical Microbiology, School of Laboratory Medicine, Beihua University, 132013 Jilin, Jilin, China \\ *Correspondence: libinxian@126.com (Binxian Li); limingcheng@beihua.edu.cn (Mingcheng Li) \\ ${ }^{\dagger}$ These authors contributed equally. \\ Academic Editor: Michael H. Dahan \\ Submitted: 20 February 2021 Revised: 6 May 2021 Accepted: 28 June 2021 Published: 11 February 2022
}

\begin{abstract}
Background: The aim of this study was to investigate the prevalence and characteristics of SCCmec genotypes and drug resistance of methicillin-resistant Staphylococcus aureus (MRSA) isolated from intensive care units (ICU) at obstetrics \& gynaecology departments in a tertiary hospital. Methods: MRSA obtained from patients admitted to the ICU were isolated and identified by using the Vitek 2 Compact System with GP21 342 cards. Antimicrobial susceptibility profiles and MRSA screening were determined by using the broth microdilution method according to CLSI guidelines. Determination of resistant genes and SCCmec genotypes were performed by multiplex PCR. Results: Of the 283 patients evaluated, 120 (42.4\%) isolates were phenotypically and genotypically confirmed to be MRSA. Among 120 strains, 15 (12.5\%) strains were SCCmec type II, 96 (80\%) strains were SCCmec type III and 9 (7.5\%) strains were undifferentiated type. All MRSA strains were recognized as multidrug resistant, exhibiting $100 \%$ resistance to cefoxitin and oxacillin, followed by erythromycin and levofloxacin (more than $80 \%$ and $90 \%$ respectively). Different SCCmec genotypes in MRAS isolates showed distinct antimicrobial agent patterns. SCCmec type II was highly resistant to clindamycin (93.3\%) with lower resistance to tetracycline (26.7\%) with SCCmec type III being highly resistant to gentamicin (91.7\%). Undifferentiated strains were resistant to Cotrimoxazole (77.8\%). There was a statistical difference among type II, type III and Undifferentiated strains $(P<0.05)$. Of interest, a high prevalence of resistance to rifampicin (more than 75\%) was also noted in the hospital. With different SCCmec genotypes, MRSA isolates were sensitive to minocycline, quinupristin, teicoplanin, vancomycin and nitrofurantoin. Conclusions: Our data indicate that SCCmec type II and SCCmec type III of MRSA are circulating in the ICU and constitute a major source for the infection spread. It is necessary to increase surveillance of MRSA in the ICU and develop adequate infection prevention strategies.
\end{abstract}

Keywords: Methicillin-resistant Staphylococcus aureus; MRSA; SCCmec; Nosocomial infection

\section{Introduction}

Methicillin-resistant Staphylococcus aureus (MRSA) is one of the most common pathogenic bacteria in intensive care units (ICU) with the majority of isolates demonstrating multidrug resistance (MDR) which impacts clinical therapy [1,2]. The resistance mechanism of MRSA is mainly secondary to the bacteria acquiring a genetic determinant (methicillin-resistant determinant $\mathrm{A}$ and $\mathrm{C}$, abbreviated as $m e c A$ or $m e c($ ), which encode penicillin-binding protein PBP2A or PBP2A'respectively [3]. These mecA and mecC genes exist in the staphylococcal cassette chromosome mec, called SCCmec. SCCmec can carry other drug resistance genes other than mec $A$ and mecC gene resulting in multiple drug resistance [4].

Emerging MRSA and multiple drug resistance are a major public health problem worldwide $[5,6]$. They are the most common cause of healthcare-associated infections (HAI) in patients that are admitted to the ICU [7]. HAI occurring in the ICU from MRSA have become particularly problematic since they arise from the treatment re- ceived by critically-ill patients [8]. Although evidence suggests a significant increase in the proportion of MRSA hospital infections worldwide, ICU at obstetrics \& gynaecology departments have reported only a limited number of MRSA isolates in China [9]. Effective and safe antimicrobial treatment is essential for treating infections in the ICU. Organizational-wide surveillance of infection-derived bacterial isolates and analysis of their susceptibility to different antimicrobial agents provides crucial information for the most effective antimicrobial therapy [10]. Furthermore, a comprehensive analysis of MRSA SCCmec typing of ICU infections and predicting the development trend of drugresistant strains is critical in evaluating disease prognosis and essential for reducing infection mortality and morbidity in the ICU.

\section{Clinical data and methods}

\subsection{Study setting and specimen collection}

This study was conducted at a university-affiliated hospital in North-East China with approximately 1200 
beds. An analysis of retrospective data of MRSA-infected patients in three obstetrics \& gynaecology ICUs was conducted during January 2018 to December 2020. A total of 283 obstetric patients were admitted after being transferred from the operating room, general ward and emergency department prior to ICU admission. Patients with concurrent HAI were classified according to infection source such as pneumonia, bloodstream infections, urinary tract infections, surgical site infections or other infections. The pathogenic bacteria associated with a HAI were collected within 48 hours of hospitalization according to the local protocol. Some samples were collected after 48 hours posthospitalization. The specimens were mainly obtained from sputum or tracheal secretions, pus, blood, ascites, catheters and drainage tubes. Multiple isolates from a single patient were excluded. The isolates from different infected sites of the same patient were also excluded. Approval for collecting clinical samples was granted by the institutional ethics committees of the participating hospital. Informed consent forms were reviewed and signed by all participants before sample collection (Ethical approval number: Protocol Number 2019-01-02).

\subsection{Identification and detection of resistance to 14 antibiotics agents}

All isolates were identified as Staphylococcus aureus by conventional standard procedures and confirmed by VITEK GNI system with GP21 342 cards (bioMérieuk Vitek Inc., Hazelwood, MO, USA). No repetitive isolates from a single patient were included. Susceptibility to 14 antimicrobial agents (Bio-Rad, Hercules, CA, USA) was determined and interpreted by the broth microdilution method akin to that in the Clinical and Laboratory Standards Institute (CLSI) criteria. MRSA screening was determined using oxacillin MIC $>6 \mu \mathrm{g} / \mathrm{mL}$ according to CLSI guidelines [11]. Control strains were Staphylococcus aureus ATCC29213, Staphylococcus aureus ATCC 25923 and Staphylococcus aureus ATCC43300, respectively.

\subsection{Determination of resistant genes and SCCmec genotypes by multiplex PCR}

A series of genes including mecA, femB, mecAa and $\mathrm{SCC} m e c$ are listed in Table 1. Multiplex PCR amplification and PCR reactions were performed as described elsewhere $[12,13]$. PCR products of genes were sent to Sangon Biotech Co., Ltd (Shanghai, China) for sequencing and DNAman software (version 6.0) (Lynnon Biosoft, Vaudreuil, QC, Canada) was used to analyse the sequencing results. Reference strains NCTC 85/2082 was used as standard strains with SCCmec type III and Reference strains NCTC N315 was used as SCCmec type II, respectively.

\subsection{Statistical analysis}

Differences in drug resistance rates of MRSA strains were analysed by Chi-square test. All drug-resistant data were analysed using SPSS version 13.0 (International Business Machines (IBM) Corp., Armonk, NY, USA). Analyses with a value of $P<0.05$ were considered to be statistically significant.

\section{Results}

\subsection{Epidemiological characteristics of specimens and isolates}

From January 2018 to December 2020, a total of 283 obstetric patients from three ICU were enrolled in this study to estimate the quantity and types of infections present in this population. The average age of the patients was $34.88 \pm 4.63$ years with a range of $17-49$ years. Thirty patients $(18.8 \%)$ were of advanced maternal age ( $\geq 35$ years). Others $(42.9 \%$ ) patients had pre-existing medical problems. Of the 283 samples, 120 were classified as SMRA by the VITEK GNI system and PCR. The remaining samples were classified as Enterobacteriaceae and nonEnterobacteriaceae, which were not included in this study (data not shown).

\subsection{Identification of mecA and femB by multiplex PCR}

Both mecA and $f e m B$ genes were identified by multiplex PCR among all MRSA isolates. The amplicons of $m e c A$ and $f e m B$ genes with a size of $310 \mathrm{bp}$ and $651 \mathrm{bp}$ were illustrated in Fig. 1A, respectively. The findings demonstrated that both mecA and $f e m B$ genes were found in all MRSA isolates obtained.

\subsection{Identification of SCCmec genotypes by multiplex PCR}

Out of 120 MRSA isolates, 15 isolates belonged to SCCmec type II (12.5\%), 96 isolates were SCCmec type III $(80 \%)$ and 9 strains belonged to an undefined type. PCR patterns are shown in Fig. $1 \mathrm{~B}$.

\subsection{Antimicrobial susceptibility analysis}

Resistance frequencies of 120 MRSA strains in this study based on CLSI microdilution demonstrated that they were recognized as multidrug resistant, exhibiting 100\% resistance to cefoxitin and oxacillin, followed by erythromycin and levofloxacin (more than $80 \%$ and $90 \%$ respectively). Different SCCmec genotypes in MRAS isolates showed distinct antimicrobial agent patterns. SCCmec type II exhibited a high incidence of resistance to clindamycin $(93.3 \%)$ but displayed a relatively low prevalence of resistance to tetracycline (26.7\%). SCCmec type III exhibited a high incidence of resistance to gentamicin $(91.7 \%)$ while undefined SCCmec types were resistant to Cotrimoxazole $(77.8 \%)$. There was a statistical difference among type II, type III and Undifferentiated strains $(P<0.05)$. No vancomycin-resistant isolate was found. Besides, MRSA isolates with different SCCmec genotypes were sensitive to minocycline, quinupristin, teicoplanin and nitrofurantoin (Table 2). 
Table 1. Oligonucleotide primers used in this study.

\begin{tabular}{|c|c|c|}
\hline Names & Primer sequences $\left(5^{\prime} \rightarrow 3^{\prime}\right)$ & Expected length (bp) \\
\hline \multirow{2}{*}{ mecA } & GTAGAAATGACTGAACGTCCGATAA & \multirow{2}{*}{310} \\
\hline & CCAATTCCACATTGATTCGGTCTAA & \\
\hline \multirow{2}{*}{$\mathrm{fem} B$} & TTACAGAGTTAACTGTTACC & \multirow{2}{*}{651} \\
\hline & ATACAAATCCAGCACGCTCT & \\
\hline \multirow{2}{*}{ mecAa } & GTGAAGATATACCAAGTGATT & \multirow{2}{*}{147} \\
\hline & ATGCGCTATAGATTGAAAGGAT & \\
\hline \multirow{2}{*}{ SCCmec I } & GCTTTAAAGAGTGTCGTTACAGG & \multirow{2}{*}{613} \\
\hline & GTTCTCTCATAGTATGACGTCC & \\
\hline \multirow{2}{*}{ SCCmec II } & CGTTGAAGATGATGAAGCG & \multirow{2}{*}{398} \\
\hline & CGAAATCAATGGTTAATGGACC & \\
\hline \multirow{2}{*}{ SCCmec III } & CCATATTGTGTACGATGCG & \multirow{2}{*}{280} \\
\hline & CCTTAGTTGTCGTAACAGATCG & \\
\hline \multirow{2}{*}{$\mathrm{SCCmec} \mathrm{IVa}$} & GCCTTATTCGAAGAAACCG & \multirow{2}{*}{776} \\
\hline & CTACTCTTCTGAAAAGCGTCG & \\
\hline \multirow{2}{*}{$\mathrm{SCCmec} \mathrm{IVb}$} & TCTGGAATTACTTCAGCTGC & \multirow{2}{*}{493} \\
\hline & AAACAATATTGCTCTCCСТC & \\
\hline \multirow{2}{*}{$\mathrm{SCCmec} \mathrm{IVc}$} & ACAATATTTGTATTATCGGAGAGC & \multirow{2}{*}{200} \\
\hline & TTGGTATGAGGTATTGCTGG & \\
\hline \multirow{2}{*}{ SCCmec IVd } & CTCAAAATACGGACCCCAATACA & \multirow{2}{*}{881} \\
\hline & TGCTCCAGTAATTGCTAAAG & \\
\hline \multirow{2}{*}{ SCCmec V } & GAACATTGTTACTTAAATGAGCG & \multirow{2}{*}{325} \\
\hline & TGAAAGTTGTACCCTTGACACC & \\
\hline
\end{tabular}
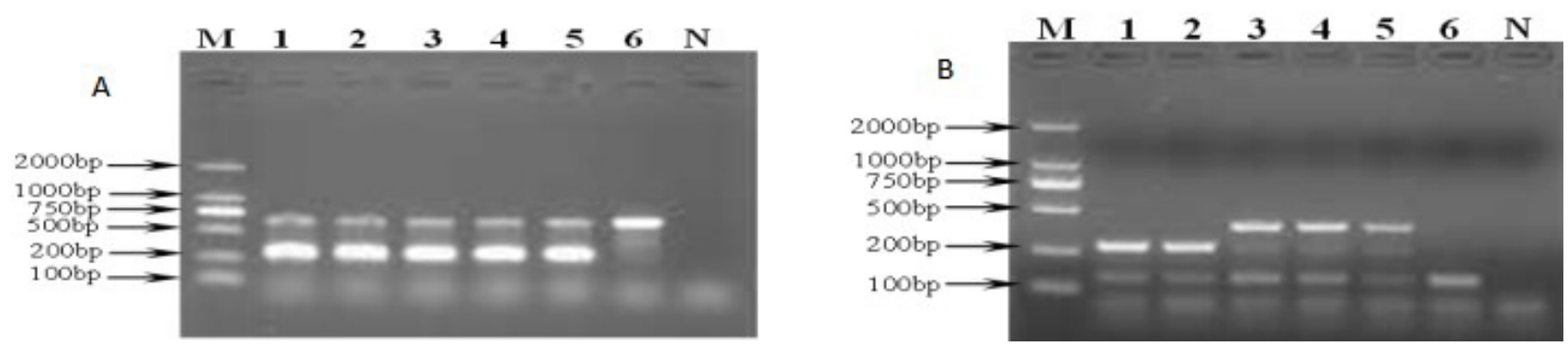

Fig. 1. Representative gel showing banding profiles by multiplex PCR analysis in MRSA isolates. (A) Agar gel electrophoresis of $m e c \mathrm{~A}$ and femB detected by multiplex PCR in MRSA isolates. M: DNA molecular weight; 1 5: MRSA isolates from different samples in ICU; 6: MSSA (ATCC25923); N: Negative control. (B) Agar gel electrophoresis of SCCmec types detected by mutiplex PCR in MRSA isolates. M: DNA molecular weight; 1: MRSA isolates SCCmec type III; 2: Reference strains 85/2082 SCCmec type III; 3: Reference strains N315 SCCmec type II; 4-5: MRSA isolates SCCmec type II; 6: MRSA isolates SCCmec undentified type; N: Negative control. MRSA, methicillin resistant Staphylococcus aureus; ICU, intensive care unit.

\section{Discussion}

Molecular typing of MRSA is an important assay for the epidemiologic investigation and strains of origin in addition to antimicrobial agent selection and therapy. Recent data demonstrate that mecA gene expresses itself in coagulase-negative staphylococcus (CNS) while $\operatorname{femB}$ is considered commonly in $S$. aureus with restricted expression but not present in CNS. Therefore, only when mecA and $f e m B$ genes are both found to be positive can MRSA be present [14]. This study amplified mecA gene (310 bp) and femB gene $(651 \mathrm{bp})$ from the $120 \mathrm{MRSA}$ strains isolated from clinical samples obtained that were determined to be MRSA.

In the form of a gene complex, mecA gene of MRSA exists in SCCmec that is composed of two gene complexes: mec gene complex and cassette chromosome recombinases (ccr). According to the structures of mec and ccr, SCCmec can be divided to five types [15]. Evidence exists that a majority of early nosocomial infections were generally considered as SCCmec type I while community-acquired MRSA infections were mostly SCCmec type IV and SCCmec type $\mathrm{V}[16,17]$. 
Table 2. Comparison of resistant characteristics of SCCmec genotypes in MRSA isolates.

\begin{tabular}{lcccc}
\hline \multirow{2}{*}{ Antimicrobial agents } & Type II $(\mathrm{n}=15)$ & Type III $(\mathrm{n}=96)$ & Type UD $(\mathrm{n}=9)$ & \multirow{2}{*}{$P$ value } \\
\cline { 2 - 4 } Cefoxitin & $\mathrm{R}(\%)$ & $\mathrm{R}(\%)$ & $\mathrm{R}(\%)$ & - \\
Oxacillin & $15(100.0)$ & $96(100.0)$ & $9(100.0)$ & - \\
Gentamicin & $15(100.0)$ & $96(100.0)$ & $9(100.0)$ & $<0.0001$ \\
Clindamycin & $0(0)$ & $88(91.7)^{*}$ & $0(0)^{\#}$ & $<0.0001$ \\
Minocycline & $14(93.3)$ & $17(17.7)^{*}$ & $3(33.3)^{*}$ & - \\
Teicoplanin & $0(0)$ & $0(0)$ & $0(0)$ & - \\
Linezolid & $0(0)$ & $0(0)$ & $0(0)$ & - \\
Quinupristin & $0(0)$ & $0(0)$ & $0(0)$ & - \\
Compound sulfamethoxazole & $0(0)$ & $0(0)$ & $0(0)$ & 1.000 \\
Erythromycin & $14(92.3)$ & $87(90.6)$ & $8(77.8)^{* \#}$ & - \\
Vancomycin & $0(0)$ & $0(0)$ & $0(0)$ & 1.000 \\
Rifampicin & $12(80.0)$ & $75(78.1)$ & $7(77.8)$ & 0.4912 \\
Levofloxacin & $14(93.3)$ & $94(97.9)$ & $9(100)$ & - \\
Nitrofurantioin & $0(0)$ & $0(0)$ & $0(0)$ & - \\
\hline
\end{tabular}

Note: ${ }^{*} P<0.05$ compared with the SCC mec type II; ${ }^{\#} P<0.05$ compared with the SCC mec type III.

$\mathrm{UD}$, undifferentiated type.

In the present study, multiplex PCR amplification was conducted to analyze characteristic genes of SCCmec genotypes. The findings showed that the predominant genotype was SCCmec type III (80\%) followed by SCCmec type II (12.5\%) while no SCCmec type IV or SCCmec type V was found among MRSA strains in the ICU. As SCCmec type IV and SCCmec type V are mainly community-acquired MRSA infections, it can be verified that communityacquired MRSA infections are not present in our ICU. This finding was in agreement with other reports that 250 clinically isolated MRSA strains were mainly SCCmec type III followed by SCCmec type II in 18 hospitals nationwide in China [18]. An explanation may be the fact that the regional difference provided disparate MRSA with a diversity of SCCmec genotypes, thus leading to distinct drug-resistant patterns. In addition, 9 (7.5\%) of 120 isolated MRSA strains of this study were undefined SCCmec strains, probably caused by the selected primers and amplification conditions or that they belong to a novel SCCmec gene, which needs further studies.

In the study, all MRSA strains were recognized as multidrug resistant. They showed a high incidence of resistance to $\beta$-lactamase, quinolone antibiotics, aminoglycosides, tetracycline and macrolides. One of the benefits from SCCmec genotyping in MRAS isolates is differentiation of antimicrobial agent susceptibility patterns. According to our findings, SCCmec type II demonstrated resistance to clindamycin $(93.3 \%)$ and tetracycline $(26.7 \%)$. SCCmec type III was strongly resistant to gentamicin $(91.7 \%)$ while undefined SCCmec types had high drug-resistance (77.8\%) to sulfamethoxazole. Due to frequent immunosuppression, patients in ICU receive more antibacterial agents than ordinary patients. Therefore, the frequencies of drug-resistant strains significantly increase in ICU compared to general wards. All isolates were sensitive to quinupristin, vancomycin and nitrofurantoin. Similar to findings reported by others $[12,19]$.

Interestingly, a high prevalence of resistance to rifampicin was noted in the hospital. In fact, rifampicin was seldom administered for the treatment of MRSA. However, the percentage of rifampicin-resistant MRSA rapidly increased from $15.5 \%$ in 2004 to $50.2 \%$ in 2008 in China. Several reported that rifampicin resistance in S. aureus isolates including MRSA was associated with mutations of $r p o \mathrm{~B}$ gene (encoding $\beta$ subunites of RNA polymerase), which conferred homogeneous methicilln resistance. However, no definitive mechanism has been elucidated to date [19-21].

The treatment of MRSA infection is a very difficult clinical problem. Risk factors for development of an MRSA infection A in the ICU include the following: widespread abuse, misuse, or overuse of antibiotics; the frequent renewal of antibiotics; increasing quantity and variety of pathogens present in the ICU; the diagnosis and progression of critical illnesses; postsurgical recovery; the use of invasive medical devices; and prolonged stay in the ICU $[22,23]$. Patients should be monitored for drug resistance within $S$. aureus so as to provide a basis for determining appropriate therapy. Meanwhile, hospitals are expected to allocate dedicated ventilators, oxygen, transfusion system, sphygmomanometer and thermometer to each patient and to disinfest the equipment after being used by each person. It is necessary to train the medical staff to infection prevention since researches verify that the hands of the medical staff are an important medium to spread MRSA, which means that hand washing is a vital way to block the spread of MRSA [22,23]. Also, attention should be paid to ventilation and air purification. Moreover, antibiotics should 
be used carefully in clinical applications to prevent drugresistant strains along with disinfection and proper isolation when indicated in order to reduce cross infection of $S$. $a u$ reus [24].

This study has a few limitations. First, genotypic or molecular data including Panton-Valentine leukocidin genes among all unidentified strains were not determined. Second, the antibiotic sensitivity of MRSA isolates to Daptomycin was not included. Future research may consider focusing on genetic types and the mechanisms for transmission.

\section{Conclusions}

The findings of this study indicate that the MRSA isolates are circulating in the ICU at obstetrics \& gynaecology departments and constitute a major source of infection at a large hospital in China. This study also found that vancomycin may be a reasonable choice in the treatment of MRSA isolates. There is a strong need for increased hospital-wide surveillance and the development of adequate infection prevention strategies.

\section{Abbreviations}

ICU, intensive care unit; CLSI, the Clinical and Laboratory Standards Institute; HAIs, healthcare-associated infections; MRSA, methicillin-resistant Staphylococcus aureus.

\section{Author contributions}

ZHH conceived, designed the experiments and wrote a draft manuscript. BXL and MCL analyzed, interpreted the results of the experiments and revised the manuscript. PYL performed the experiments. XM collected the clinical data. All authors read and approved the final manuscript.

\section{Ethics approval and consent to participate}

Ethical approval for collecting clinical samples was received by the institutional ethics committees of the participating hospital. Informed consent forms were reviewed and signed by all participants before samples collection (Ethical approval number: Protocol Number 2019-01-02).

\section{Acknowledgment}

We thank three anonymous reviewers for excellent criticism of the article.

\section{Funding}

The present project was a part of National Natural Science Foundation of China project (81402979), the Jilin Science and Technology Development Program (20190304101YY and 20190301014NY); the Health and Family Planning Commission of Jilin Province (2018J098) also supported the project.

\section{Conflict of interest}

The authors declare no conflict of interest.

\section{References}

[1] Stefani S, Chung DR, Lindsay JA, Friedrich AW, Kearns $\mathrm{AM}$, Westh $\mathrm{H}$, et al. Meticillin-resistant Staphylococcus aureus (MRSA): global epidemiology and harmonisation of typing methods. International Journal of Antimicrobial Agents. 2012; 39: 273-282.

[2] Bouras D, Doudoulakakis A, Tsolia M, Vaki I, Giormezis N, Petropoulou N, et al. Staphylococcus aureus osteoarticular infections in children: an 8-year review of molecular microbiology, antibiotic resistance and clinical characteristics. Journal of Medical Microbiology. 2018; 67: 1753-1760.

[3] Sutter DE, Milburn E, Chukwuma U, Dzialowy N, Maranich AM, Hospenthal DR. Changing Susceptibility of Staphylococcus aureus in a us Pediatric Population. Pediatrics. 2016; 137: e20153099.

[4] Hetem DJ, Derde LPG, Empel J, Mroczkowska A, Orczykowska-Kotyna M, Kozińska A, et al. Molecular epidemiology of MRSA in 13 ICUs from eight European countries. Journal of Antimicrobial Chemotherapy. 2016; 71: $45-52$.

[5] Rajan V, Schoenfelder SMK, Ziebuhr W, Gopal S. Genotyping of community-associated methicillin resistant Staphylococcus aureus (CA-MRSA) in a tertiary care centre in Mysore, South India: ST2371-SCCmec IV emerges as the major clone. Infection, Genetics and Evolution. 2015; 34: 230-235.

[6] Aggarwal S, Jena S, Panda S, Sharma S, Dhawan B, Nath G, et al. Antibiotic susceptibility, virulence pattern, and typing of Staphylococcus aureus strains isolated from variety of infections in India. Frontiers in Microbiology. 2019; 10: 2763.

[7] Erikstrup LT, Dinh KM, Andersen PS, Skov RL, Kaspersen KA, Nielsen KR, et al. Cohort description: the Danish Blood Donor Staphylococcus aureus Carriage Study. Clinical Epidemiology. 2019; 11: 885-900.

[8] Rodríguez EA, Correa MM, Ospina S, Atehortúa SL, Jiménez $\mathrm{JN}$. Differences in epidemiological and molecular characteristics of nasal colonization with Staphylococcus aureus (MSSAMRSA) in children from a university hospital and day care centers. PLoS ONE. 2014; 9: e101417.

[9] Jian Y, Zhao L, Zhao N, Lv H, Liu Y, He L, et al. Increasing prevalence of hypervirulent ST5 methicillin susceptible Staphylococcus aureus subtype poses a serious clinical threat. Emerging Microbes \& Infections. 2021; 10: 109-122.

[10] Hemmadi V, Biswas M. Dramatic Changes in Oligomerization Property Caused by Single Residue Deletion in Staphylococcus aureus Enolase. Molecular Biotechnology. 2021; 63: 125-139.

[11] Clincal and Laboratory Standards Institute. Performance Standards for Antimicrobial Susceptibility Testings; Nineteeth Informational Supplement. PA, USA: M1O0-S19-Wayne. 2012.

[12] Sara AO, Ariadnna CC, Jetsi MR, Gerardo EV, Veronica EK, Isabel FH, et al. Control of Methicillin-Resistant Staphylococcus aureus Strains Associated With a Hospital Outbreak Involving Contamination From Anesthesia Equipment Using UV-C. Frontiers in Microbiology. 2020; 11: 600093.

[13] Chao G, Zhang X, Zhang X, Huang Y, Xu L, Zhou L, et al. Phenotypic and genotypic characterization of methicillin-resistant Staphylococcus aureus (MRSA) and methicillin-susceptible Staphylococcus aureus (MSSA) from different sources in China. Foodborne Pathogens and Disease. 2013; 10: 214-221.

[14] Kim J, Kim BE, Ahn K, Leung DYM. Interactions between atopic dermatitis and Staphylococcus aureus infection: clinical implications. Allergy, Asthma \& Immunology Research. 2019; 11: 593-603. 
[15] Challagundla L, Reyes J, Rafiqullah I, Sordelli DO, EchanizAviles G, Velazquez-Meza ME, et al. Phylogenomic Classification and the Evolution of Clonal Complex 5 MethicillinResistant Staphylococcus aureus in the Western Hemisphere. Frontiers in Microbiology. 2018; 9: 1901.

[16] Goudarzi M, Seyedjavadi SS, Nasiri MJ, Goudarzi H, Sajadi Nia R, Dabiri H. Molecular characteristics of methicillin-resistant Staphylococcus aureus (MRSA) strains isolated from patients with bacteremia based on MLST, SCCmec, spa, and agr locus types analysis. Microbial Pathogenesis. 2017; 104: 328-335.

[17] Ranjbar R, Moazzami Goudarzi M, Jonaidi N, Moeini R. Cassette chromosome mec typing of methicillin-resistant Staphylococcus aureus isolates from patients in Tehran. Molecular Genetics, Microbiology and Virology. 2016; 31: 109-115.

[18] Dai Y, Liu J, Guo W, Meng H, Huang Q, He L, et al. Decreasing methicillin-resistant Staphylococcus aureus (MRSA) infections is attributable to the disappearance of predominant MRSA ST239 clones, Shanghai, 2008-2017. Emerging Microbes \& Infections. 2019; 8: 471-478.

[19] Wichelhaus TA, Schäfer V, Brade V, Böddinghaus B. Molecular characterization of rpoB mutations conferring cross-resistance to rifamycins on methicillin-resistant Staphylococcus aureus.
Antimicrobial Agents and Chemotherapy. 1999; 43: 2813 2816.

[20] Murugan K, Kavitha K, Al-Sohaibani S. Rifampicin resistance among multi-resistant MRSA clinical isolates from Chennai, India, and their molecular characterization. Genetics and Molecular Research. 2015; 14: 2716-2725.

[21] Zhou W, Shan W, Ma X, Chang W, Zhou X, Lu H, et al. Molecular characterization of rifampicin-resistant Staphylococcus aureus isolates in a Chinese teaching hospital from Anhui, China. BMC Microbiology. 2012; 12: 240.

[22] Gibson CV. Emergency medical services oxygen equipment: a fomite for transmission of MRSA? Emergency Medicine Journal. 2019; 36: 89-91.

[23] Urushibara N, Aung MS, Kawaguchiya M, Kobayashi N. Novel staphylococcal cassette chromosome mec (SCCmec) type XIV (5a) and a truncated SCCmec element in SCC composite islands carrying speG in ST5 MRSA in Japan. Journal of Antimicrobial Chemotherapy. 2020; 75: 46-50.

[24] Harris SR, Feil EJ, Holden MTG, Quail MA, Nickerson EK, Chantratita N, et al. Evolution of MRSA during Hospital Transmission and Intercontinental Spread. Science. 2010; 327: 469474. 